\title{
ON THE POSSIBILITIES OF DETECTING DARK MATTER BY THE
} GRAVITATIONAL LENS EFFECT

\author{
R. Kayser \\ Hamburger Sternwarte \\ Gojenbergsweg 112 \\ D-2050 Hamburg 80 \\ F.R. Germany
}

A class of compact objects with cosmological density leads to a probability for multiple imaging by gravitational lensing of roughly $P \approx \rho / \rho_{c}$, where $\rho_{c}$ is the critical density.

Thus every kind of compact objects which contribute significantly to $\Omega$ should be detectable by lens effects. This is of special interest for the lower end of the mass spectrum, i.e. for the detection of brown dwarves and jupiters ("micro-lenses"). Gravitational micro-lensing does not lead to observable separations of quasar images, but may induce variability to the quasar brightness. However, the question is : How to distinguish between variability induced by micro-lensing and intrinsic variability ?

If the time delay between two macro-images is known the problem is trivial, since only intrinsic variations appear ( $t$ ime-shifted) in both images. If the time delay is not known or no multiple imaging occurs, the problem may be solved by

1. typical lightcurves ${ }^{1)}$

If the compact continuum source crosses an anti-caustic, a typical asymmetric maximum appears in the light curve.

2. spectral effects ${ }^{1)}$

The influence of micro-lensing depends on the source size.

3. parailax effects ${ }^{2)}$ 3)

micro-lensing leads to a brightness gradient at the observer, which should be observable by interplanetary probes even with relative small telescopes.

Ref.: 1) Kayser,Refsdal,Stabell: 1986,Astron.Astroph.166,36

2) Grieger,Kayser,Refsdal: 1986, Nature 324, 126

3) Grieger,Kayser,Refsdal: 1987, subm. Astron.Astroph.

601

J. Audouze et al. (eds.), Large Scale Structures of the Universe, 601 .

(c) 1988 by the IAU. 